\title{
Ventilação Mecânica na Doença Pulmonar Obstrutiva Crônica
}

\section{Mechanical Ventilation in Chronic Obstructive Pulmonary Disease}

\author{
Sérgio Jezler ${ }^{1}$, Marcelo A.Holanda ${ }^{2}$, Anderson José ${ }^{3}$, Suelene Aires Franca ${ }^{4}$
}

\section{RESUMO}

JUSTIFICATIVA E OBJETIVOS: Em 2000, foi publicado o II Consenso Brasileiro de Ventilação Mecânica. Desde então, o conhecimento na área da ventilação mecânica avançou rapidamente, com a publicação de numerosos estudos clínicos que acrescentaram informações importantes para o manejo de pacientes críticos em ventilação artificial. Além disso, a expansão do conceito de Medicina Baseada em Evidências determinou a hierarquização das recomendações clínicas, segundo o rigor metodológico dos estudos que as embasaram. Essa abordagem explícita vem ampliando a compreensão e a aplicação das recomendações clínicas. Por esses motivos, a AMIB - Associação de Medicina Intensiva Brasileira - e a SBPT - Sociedade Brasileira de Pneumologia e Tisiologia - julgaram conveniente a atualização das recomendações descritas no Consenso anterior. Dentre os tópicos selecionados a Ventilação Mecânica na Agudização da DPOC foi um dos temas propostos. O objetivo deste estudo foi descrever os pontos mais importantes relacionados à ventilação mecânica durante a agudização da doença pulmonar obstrutiva crônica (DPOC) e sugerir as principais abordagens terapêuticas.

MÉTODO: Objetivou-se chegar a um documento suficientemente sintético, que refletisse a melhor evidência disponível na literatura. A revisão bibliográfica baseou-se na busca de estudos através de palavraschave e em sua gradação conforme níveis de evidência. As palavras-chave utilizadas para a busca foram: ventilação mecânica na DPOC: COPD and mechanical ventilation.

\footnotetext{
1. Faculdade de Medicina da Universidade Federal da Bahia (BA)

2. Faculdade de Medicina da Universidade Federal do Ceará (CE)

3. UTI da Santa Casa de São Paulo (SP)

4. UTI do Hospital das Clinicas da Universidade Federal de Goiás (GO)

(C)Associação de Medicina Intensiva Brasileira, 2007
}

RESULTADOS: São apresentadas recomendações quanto aos modos ventilatórios e aos parâmetros a serem aplicados quando do ajuste do ventilador, além da monitoração recomendada. Apresentam-se ainda, técnicas alternativas que possam ser utilizadas.

CONCLUSÕES: Estratégias protetoras de ventilação mecânica são recomendadas durante a ventilação mecânica de um paciente DPOC agudizado.

Unitermos: agudização da DPOC, Consenso, recomendação, ventilação mecânica

\section{SUMMARY}

BACKGROUND AND OBJECTIVES: The II Brazilian Consensus Conference on Mechanical Ventilation was published in 2000. Knowledge on the field of mechanical ventilation evolved rapidly since then, with the publication of numerous clinical studies with potential impact on the ventilatory management of critically ill patients. Moreover, the evolving concept of evidence - based medicine determined the grading of clinical recommendations according to the methodological value of the studies on which they are based. This explicit approach has broadened the understanding and adoption of clinical recommendations. For these reasons, AMIB - Associação de Medicina Intensiva Brasileira and SBPT - Sociedade Brasileira de Pneumologia e Tisiologia - decided to update the recommendations of the II Brazilian Consensus. Mechanical ventilation in COPD exacerbation has been one of the updated topics. Describe the most important topics on the mechanical ventilation during the COPD exacerbation and suggest the main therapeutic approaches.

METHODS: Systematic review of the published literature and gradation of the studies in levels of evidence, using the keywords "mechanical ventilation" and "COPD".

RESULTS: We present recommendations on the ventilatory modes and settings to be adopted when ven- 
tilating a patient during an asthma attack, as well as the recommended monitoring. Alternative ventilation techniques are also presented.

CONCLUSIONS: Protective ventilatory strategies are recommended when ventilating a patient during a.

Key Words: Consensus, COPD, mechanical ventilation, recommendation.

\section{INTRODUÇÃO}

A sigla DPOC (Doença Pulmonar Obstrutiva Crônica) denomina um grupo de entidades nosológicas respiratórias que acarretam obstrução crônica ao fluxo aéreo de caráter fixo ou parcialmente reversível, tendo como alterações fisiopatológicas de base, graus variáveis de bronquite crônica e enfisema pulmonar ${ }^{1}$. Sob o tópico de DPOC não se enquadram pacientes portadores de: bronquiectasias difusas, seqüelas de tuberculose, asma, bronquiolites, pneumoconioses ou outras doenças parenquimatosas pulmonares ${ }^{1,2}$.

\section{EPIDEMIOLOGIA}

Estima-se que 5,5 milhões de pessoas sejam acometidas por DPOC no Brasil ${ }^{1}$ e 52 milhões em todo o mundo, tendo sido essa doença responsável por 2,74 milhões de óbitos em $2000^{2}$. No Brasil, vem ocupando entre a $4^{a}$ e $7^{a}$ posição entre as principais causas de morte ${ }^{1}$. Nos EUA, é a $4^{a}$ causa mais freqüente. Nos dois países a taxa de mortalidade é crescente. No Brasil, no ano de 2003, a DPOC foi a $5^{\text {a }}$ maior causa de hospitalização de pacientes maiores de 40 anos no sistema público de saúde (cobertura de $80 \%$ da população), com 196.698 internações e gasto aproximado de 72 milhões de reais ${ }^{1}$.

As exacerbações são freqüentes (2,4 a 3 episódios/ ano por paciente). Em cerca de $50 \%$ das vezes o paciente não procura o médico. Em casos de internação, a mortalidade hospitalar geral é de $3 \%$ a $4 \%$, podendo alcançar de $11 \%$ a $24 \%$, quando há necessidade de tratamento intensivo, e chegando a atingir de $43 \%$ a $46 \%$ em um ano. A probabilidade de reinternação por nova exacerbação é de $50 \%$ em 6 meses. Após uma exacerbação, é de se esperar redução temporária da função pulmonar e da qualidade de vida. Vale ressaltar que, em recente estudo, cerca de $3 / 4$ dos pacientes admitidos em hospital por exacerbação de DPOC que necessitaram de intubação traqueal sobreviveram e que cerca da metade dos pacientes continuaram vivos após dois anos da admissão ${ }^{2-4}$.

\section{FISIOPATOLOGIA}

O desenvolvimento ou agravamento da hiperinsuflação pulmonar dinâmica, com aprisionamento aéreo, consiste na principal alteração fisiopatológica na exacerbação da DPOC. Os principais mecanismos envolvidos são: aumento da obstrução ao fluxo aéreo (causada por inflamação, hipersecreção brônquica e broncoespasmo) acompanhado de redução da retração elástica pulmonar. Todos esses fatores resultam em prolongamento da constante de tempo expiratória, ao mesmo tempo em que se eleva a freqüência respiratória como resposta ao aumento da demanda ventilatória, encurtando-se o tempo para expiração. A hiperinsuflação dinâmica gera aumento substancial da auto-PEEP ou PEEP intrínseca (PEE$\mathrm{Pi}$ ), impondo uma sobrecarga de trabalho à musculatura inspiratória para deflagração de fluxo de ar na inspiração. Por sua vez, a hiperinsuflação também compromete a performance muscular respiratória, modificando a conformação geométrica das fibras musculares, reduzindo a curvatura diafragmática. Além disso, nos pacientes com doença mais avançada, pode haver diminuição direta da força muscular por uso crônico de corticosteróides e desnutrição. Nas exacerbações muito graves, pode haver diminuição da resposta do comando neural (drive) no centro respiratório à hipóxia e à hipercapnia, estas decorrentes do desequilíbrio ventilação/perfusão e de hipoventilação alveolar, agravando a acidose respiratória e a hipoxemia arterial ${ }^{5}$.

\section{INDICAÇÕES DA VENTILAÇÃO MECÂNICA INVASIVA}

O suporte ventilatório mecânico na DPOC está indicado nas exacerbações com hipoventilação alveolar e acidemia e, menos freqüentemente, naquelas com hipoxemia grave não corrigida pela oferta de oxigênio suplementar ${ }^{1}$. Embora a ventilação mecânica não-invasiva (VMNI) seja o suporte ventilatório de escolha na exacerbação da DPOC, sua utilização é possível em número limitado de pacientes selecionados. Aqueles com apresentação mais grave em geral necessitam intubação traqueal com ventilação invasiva, o mesmo procedimento ocorrendo em casos de falha da VMNI (Quadro 1) ) $^{2,5,6}$. Em estudo retrospectivo, 54\% de 138 pacientes com DPOC descompensada, cursando com hipercapnia necessitaram de intubação traqueal, em média, oito horas após a apresentação ${ }^{7}$. 
Quadro 1 - Situações Clínicas em que a Intubação Traqueal e Ventilação Mecânica Invasiva são Preferíveis ao Uso de Ventilação Mecânica Não-Invasiva na Exacerbação da DPOC

Parada cardiorrespiratória iminente
Depressão do nível de consciência *
Agitação psicomotora
Pneumonia associada
Obstrução de vias aéreas superiores
Excesso de secreção
Reflexo de tosse comprometido
Doenças concomitantes (encefalopatia grave, hemorragia di-
gestiva alta, instabilidade hemodinâmica, disritmias cardíacas)

*Alguns autores consideram pacientes com narcose e hipercapnia candidatos a uma tentativa com VMNI antes de se proceder à intubação.

\section{OBJETIVOS DA VENTILAÇÃO MECÂNICA}

Ao se instituir a ventilação mecânica invasiva na DPOC exacerbada os seguintes objetivos terapêuticos devem nortear a estratégia ventilatória (Quadro 2)5:

Quadro 2 - Objetivos Terapêuticos Relacionados à Ventilação Mecânica Invasiva na DPOC

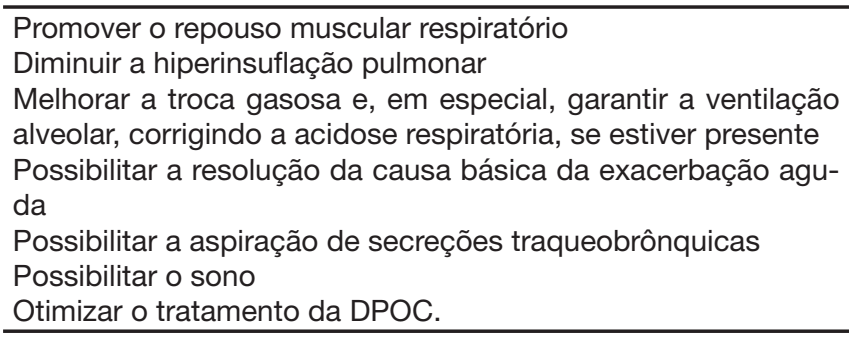

\section{AJUSTE INICIAL DA VENTILAÇÃO MECÂNICA (Quadro 3)}

Quadro 3 - Graus de Recomendação

Graus de Re- Tratamento/Prevenção comendação

A

$\mathrm{B}$

C Relato de casos (incluindo coorte ou casocontrole de menor qualidade)

D Opinião de especialista sem avaliação crítica ou baseada em matérias básicas (estudo fisiológico ou estudo com animais)

\section{Promoção do Repouso Muscular Respiratório}

Recomendação: Promover repouso muscular respiratório, viabilizando a reversão da fadiga muscular através da utilização de modo ventilatório assistidocontrolado, acompanhado de sedação e analgesia tituladas para supressão ou grande diminuição da atividade muscular respiratória, nas primeiras 24 a 48 horas. Sedação e analgesia adequadas são também importantes para a redução da produção de $\mathrm{CO}_{2}$, o que, por si só, já auxilia na redução da demanda ventilatória.

\section{Grau de Recomendação: D}

Comentário: Embora não haja ensaios clínicos controlados avaliando essa estratégia ventilatória, sua aplicação leva em conta que pacientes em exacerbação de DPOC realizam intenso trabalho respiratório, encontrando-se em franca ou no limiar de fadiga muscular ${ }^{5}$. O tempo de repouso recomendado baseia-se em estudos que demonstraram que, pelo menos 24 horas, seriam necessárias para completa reversão da fadiga muscular respiratória ${ }^{8}$. O controle ventilatório total permite também a adoção de estratégias ventilatórias "não-fisiológicas" como a hipoventilação controlada com hipercapnia permissiva. Não raro, pacientes com DPOC apresentam cardiopatias associadas, de modo que a supressão do esforço muscular respiratório pode trazer vantagens do ponto de vista hemodinâmico, otimizando a relação entre transporte, consumo e extração de oxigênio. Uma outra vantagem da ventilação controlada é possibilitar a mensuração precisa da mecânica pulmonar (medidas da pressão de platô e da auto-PEEP, valores que, juntamente com a pressão de pico, taxa de fluxo inspiratório e volume-corrente, permitem os cálculos da resistência, complacência e constante de tempo do sistema respiratório) ${ }^{9}$.

\section{MODO DE CONTROLE DA FASE INSPIRATÓRIA}

Recomendação: Não há estudo comparativo entre os modos volume-controlado (ciclado a volume) ou pressão controlada (ciclado a tempo) em pacientes com exacerbação da DPOC. Independente do modo de controle selecionado, as estratégias ventilatórias visando ao repouso muscular respiratório e à minimização da hiperinsuflação pulmonar dinâmica, deve ser priorizadas.

\section{Grau de Recomendação: D}

Comentário: Na ventilação com pressão controlada, os seguintes ajustes são determinantes indiretos do volume-minuto (VE) final e, portanto, do grau de hi- 
perinsuflação dinâmica: o tempo inspiratório (TI); a variação de pressão inspiratória - pressão motriz ou driving pressure (pressão inspiratória programada menos a PEEP total) -; e a freqüência respiratória (FR). O nível programado de pressão inspiratória, o TI e a impedância do sistema respiratório determinarão indiretamente o volume-corrente (VT) e o fluxo inspiratório. Em uma situação de instabilidade da mecânica pulmonar com variações de impedância (complacência do sistema respiratório, resistência das vias aéreas e auto-PEEP) podem ocorrer grandes variações do VT e do fluxo inspiratório e, conseqüentemente, dos níveis de $\mathrm{pH}$ arterial, $\mathrm{PaCO}_{2}$ e de insuflação pulmonar. É imprescindível a monitoração do VE e do VT a fim de se detectar prontamente tais variações, quando em uso desse modo de controle.

$\mathrm{Na}$ ventilação com volume-controlado, os fatores determinantes do VE são ajustados diretamente no ventilador: VT; fluxo inspiratório; e freqüência respiratória. É possível manter-se um VE estável, prevenindo-se grandes flutuações da ventilação alveolar, porém existe o risco de liberação de altas pressões inspiratórias em casos de aumentos da impedância do sistema respiratório, fato que torna a monitoração dessas pressões obrigatória. As medidas de mecânica pulmonar (notadamente pressão de platô e resistência de vias aéreas) são facilitadas no modo ciclado a volume. Já na ventilação com pressão controlada, tem-se um risco menor de se causar hiperdistensão de alvéolos com melhor complacência, fato que poderia evitar eventual lesão pulmonar induzida pelo ventilador ${ }^{10,11}$.

\section{AJUSTES PARA DIMINUIÇÃO DA HIPERINSUFLA- ÇÃO PULMONAR}

Todo o cuidado deve ser tomado visando à diminuição da hiperinsuflação pulmonar e suas complicações: repercussão hemodinâmica; barotrauma; e aumento do trabalho respiratório ${ }^{1}$. Nesses pacientes, é importante a monitorização gráfica da curva de fluxo expiratório pelo tempo: um fluxo expiratório lento, que persiste até o início da próxima inspiração é um marco da presença de hiperinsuflação dinâmica ${ }^{12}$.

\section{VOLUME-MINUTO (VE) E VENTILAÇÃO ALVEOLAR}

Recomendação: $A$ redução do volume-minuto (VE = VT x FR), através da utilização de baixo volume-corrente e/ou baixa freqüência respiratória, é a estratégia ventilatória mais eficiente visando à redução da hiperinsuflação dinâmica. Os ajustes do volume-minuto (sobretudo da freqüência respiratória) devem ter por meta a manutenção de um pH arterial entre 7,20 e 7,40, independente da $\mathrm{PaCO}_{2}$ (desde que não haja situações potencialmente mais graves na vigência de hipercapnia, como disritmias, hipertensão intracraniana ou insuficiência coronariana). A freqüência respiratória deve ser a menor possível, atingidos os objetivos acima, tolerando-se valores inferiores a 10-12 irpm. Recomenda-se um volume-corrente em torno de 6 a $8 \mathrm{~mL} / \mathrm{kg}$, a fim de se reduzir ao máximo o tempo inspiratório (TI) e prolongar a expiração ${ }^{13-17}$.

\section{Grau de Recomendação: D}

Comentário: Tentativas para correção da hipercapnia visando à redução da $\mathrm{PaCO}_{2}$, tendo por meta sua normalização (valores abaixo de $45 \mathrm{mmHg}$ ), podem resultar em alcalose sangüínea, com seus efeitos negativos sobre a oferta tissular de oxigênio, depressão do sistema nervoso central com potencial redução do fluxo sangüíneo para o encéfalo e hipotensão ${ }^{18,19}$. Além disso, valores supranormais de $\mathrm{PaCO}_{2}$ são compatíveis com ajustes de baixos volumes-minuto o que, por sua vez, consiste na principal medida para diminuir a hiperinsuflação pulmonar. Não há ensaios controlados comparando diferentes níveis de VT.

\section{FLUXO INSPIRATÓRIO}

Recomendação: Não há diferenças clínicas relevantes entre a administração de um fluxo quadrado (constante) ou em rampa (desacelerado) ${ }^{1,2}$. No modo pressão-controlada, o fluxo é livre, porém, no modo volume controlado, a escolha dos valores de fluxo devem levar em conta os tempos ins (TI) e expiratório (TE), ou seja, o volume-corrente e a freqüência respiratória (FR). Na ventilação com volume controlado, recomenda-se inicialmente o uso de fluxo quadrado ou constante como opção inicial em valores que otimizem a relação I:E através do controle do $\mathrm{Tl}$, o que em geral corresponde a taxas que variam entre 40 e $80 \mathrm{~L} / \mathrm{min}$ ou 5 e 6 vezes o volume-minuto (VE).

\section{Grau de Recomendação: D}

Comentário: A função de fluxo desacelerado varia conforme o ventilador utilizado. A depender do aparelho, uma mudança no padrão de fluxo pode resultar em: a) manutenção do TI, elevando-se o fluxo inicial e produzindo sua redução até zero (fluxo médio igual nos dois padrões) ou b) prolongamento do $\mathrm{TI}$, se o pico de fluxo inicial no padrão desacelerado for o 
mesmo do padrão constante (quadrado) com redução linear ao longo da inspiração. O fluxo desacelerado promove redução do pico de pressão inspiratória sem outras repercussões na mecânica pulmonar e pode resultar em menor ventilação de espaço morto, calculado pela relação VD/VT, com redução da $\mathrm{PaCO}_{2}$. Por outro lado, diminuições do fluxo ins com o único intuito de se reduzir a pressão de pico prolongam o $\mathrm{Tl}$ e têm o potencial de agravarem o aprisionamento aéreo e a auto-PEEP ${ }^{20,21}$. A medida da resistência inspiratória de vias aéreas pelo método de oclusão ao final da inspiração não é acurada com o fluxo desacelerado. Na ventilação com pressão controlada, o fluxo inspiratório dependerá do gradiente pressórico entre a pressão programada e a pressão alveolar, da impedância do sistema respiratório (complacência e resistência, ou seja, da constante de tempo) e do tempo inspiratório programado. A monitoração das curvas de fluxo, volume e pressão versus tempo podem facilitar o ajuste neste modo.

\section{TEMPO INSPIRATÓRIO (TI), TEMPO EXPIRATÓRIO (TE) E RELAÇÃO I:E}

Recomendação: Recomenda-se uma relação I:E inferior a 1:3 (isto é, 1:4, 1:5, etc.), em situação de baixa freqüência respiratória com tempo expiratório prolongado o suficiente para redução do aprisionamento aéreo e da auto-PEEP ao máximo. No modo volume controlado, o tempo expiratório pode ser prolongado pelo uso de altas taxas de fluxo inspiratório e pela não utilização de pausa inspiratória de forma rotineira. No modo pressão controlada, ajusta-se diretamente no ventilador um tempo inspiratório mais curto. Seguindo os ajustes sugeridos, a maioria dos pacientes será ventilada com relações I:E inferiores ou próximas de 1:5 a 1:8 (exemplo de ajuste do modo volume controlado em um paciente de $70 \mathrm{~kg}: \mathrm{V}_{\mathrm{T}}=500 \mathrm{Ml}$; Fluxo $=60 \mathrm{~L} / \mathrm{min}$, quadrado; $\mathrm{FR}=12 \mathrm{irpm} ; \mathrm{TI}=0,5 \mathrm{~s} ; \mathrm{TE}=$ $4,5 \mathrm{~s} ; \rightarrow$ relação I:E = 1:9).

\section{Grau de Recomendação: D}

Comentário: A obstrução ao fluxo aéreo e a elevada resistência de vias aéreas, sobretudo na expiração, tornam necessário um tempo expiratório prolongado para garantir o esvaziamento alveolar. Assim, procura-se reduzir o TI e aumentar o TE ao máximo, diminuindo a relação I:E, para redução da hiperinsuflação dinâmica ${ }^{1}$. Os parâmetros ventilatórios iniciais podem ser reajustados após as medidas de mecânica pulmonar serem obtidas. A constante de tempo do sistema respiratório (produto da resistência pela compla- cência) determina a taxa de esvaziamento pulmonar passivo. Admite-se que três constantes de tempo são necessárias para reduzir passivamente o volume pulmonar a $5 \%$ de seu valor inicial. De posse deste conhecimento, alguns cálculos simples podem ajudar a reajustar individualmente os parâmetros ventilatórios em um paciente com DPOC. Por exemplo, em um paciente com uma resistência de vias aéreas de $20 \mathrm{cmH}_{2} \mathrm{O} / \mathrm{L} / \mathrm{s}$ e uma complacência do sistema respiratório de $0,05 \mathrm{~L} / \mathrm{cmH}_{2} \mathrm{O}$, uma fase expiratória de 3 s $(3 \times 20 \times 0,05)$ será necessária para a exalação passiva de $95 \%$ do volume-corrente inspirado. Isso poderia ser conseguido com uma freqüência respiratória de 15 irpm e uma I:E de 1:3. A mesma freqüência respiratória com uma I:E maior (por exemplo, 1:2) seria insuficiente para promover esvaziamento completo, visto que o tempo expiratório seria de 2,66 s. Da mesma forma, com uma freqüência respiratória de 20 irpm nunca se conseguiria atingir este objetivo, visto que a duração total do ciclo respiratório seria de 3 segundos 22 .

\section{OXIGENAÇÃO, FRAÇÃO INSPIRADA DE OXIGÊNIO $\left(\mathrm{FIO}_{2}\right)$}

Recomendação: $\mathrm{A} \mathrm{FIO}_{2}$ deve ser titulada evitando-se a hiperóxia $\left(\mathrm{PaO}_{2}>120 \mathrm{mmHg}\right)$, preferindo-se garantir valores de $\mathrm{PaO}_{2}$ mais próximos do limite inferior da faixa terapêutica (60 a $80 \mathrm{mmHg}$ ), desde que se garanta uma $\mathrm{SaO}_{2}$ superior a $90 \%$. Taxas de $\mathrm{SaO}_{2}$ nesta faixa não comprometem a oxigenação tissular desde que não haja comprometimento do débito cardíaco e da concentração de hemoglobina.

\section{Grau de Recomendação: D}

Comentário: Taxas elevadas de $\mathrm{FIO}_{2}$ podem acentuar o desequilíbrio ventilação-perfusão (V'A/Q') e agravar a hipercapnia e a acidose respiratória na DPOC, através de aumento da relação entre o espaço morto e o volume-corrente (VD/VT). Em geral os pacientes com DPOC exacerbada não necessitam elevadas $\mathrm{FIO}_{2}$ para a reversão da hipoxemia, excetuando-se os quadros clínicos em que há doença parenquimatosa com mecanismos de shunt pulmonar associada, tais como pneumonia extensa ou edema alveolar. Considera-se também que qualquer agravamento adicional da acidose respiratória, ocasionada pelo uso de altas $\mathrm{FIO}_{2}$ $\left(\mathrm{FIO}_{2}>0,4\right)$, pode induzir a reajustes da ventilação mecânica visando ao aumento do V'E, com potencial agravamento da hiperinsuflação dinâmica. Por outro lado, estudo prospectivo e encoberto não evidenciou indução de hipercapnia, acidose ou elevação do es- 
paço morto após elevação por 20 minutos da $\mathrm{FIO}_{2}$ de $30 \%$ ou $40 \%$ para $70 \%$ em 12 pacientes com DPOC em fase de desmame da $\mathrm{VM}^{23}$.

\section{MONITORIZAÇÃO DA MECÂNICA PULMONAR}

Recomendação: Devem ser medidos de forma rotineira o pico de pressão, a pressão de platô e a resistência de vias aéreas.

\section{Grau de Recomendação: D}

Comentário: Os valores de pico de pressão ou pressão inspiratória máxima não devem ser levados em conta isoladamente para mudanças na estratégia ventilatória, pois não se correlacionam com a pressão alveolar em pacientes com resistência elevada de vias aéreas. Por outro lado, picos de pressão superiores a $45 \mathrm{cmH}_{2} \mathrm{O}$ sinalizam para problemas potencialmente graves envolvendo a mecânica pulmonar e exigem rápida avaliação diagnóstica e tratamento. A pressão de pausa ou de platô inspiratório correlaciona-se com o grau de hiperinsuflação pulmonar. Recomenda-se sua monitoração sistemática limitando-a a valores inferiores a $30 \mathrm{cmH}_{2} \mathrm{O}$. A resistência de vias aéreas pode ser estimada dividindo-se a diferença entre a pressão de pico e a pressão de platô pelo fluxo inspiratório constante (onda quadrada). Os valores encontrados podem orientar a instituição ou avaliar eficácia da terapêutica broncodilatadora. No caso da ventilação com pressão controlada, devem ser monitorados o V'E e o VT expirado ${ }^{12}$.

\section{PRESSÕES EXPIRATÓRIAS: PEEP EXTRÍNSECA E AUTO-PEEP}

Recomendação: A PEEP extrínseca (PEEPe) pode ser utilizada nos pacientes portadores de DPOC, para contrabalançar a PEEP intrínseca (PEEPi) secundária à limitação ao fluxo aéreo expiratório. Embora ainda não existam dados suficientes para uma recomendação específica sobre o ajuste do seu valor ideal, o uso de PEEPe em valores próximos aos da PEEPi (estática) mensurada (não maior que $85 \%$ dela), parece reduzir o trabalho respiratório sem causar aumento da hiperinsuflação e do volume pulmonar expiratório final. Visto que os efeitos da PEEPe não são sempre reprodutíveis em todos os pacientes, variações do volume pulmonar expiratório final devem ser monitoradas a fim de se evitar piora da hiperinsuflação dinâmica. Para isso, a medida da pressão de pausa (platô inspiratório) é recomendada e pode ser usada para titulação da PEEPe, objetivando-se manter valores inferiores a $30 \mathrm{cmH}_{2} \mathrm{O}$. A aplicação da PEEPe deve le- var em conta a PEEPi medida após a otimização dos ajustes iniciais.

\section{Grau de Recomendação: D}

Comentário: Hiperinsuflação dinâmica e a presença de PEEPi são observadas em pacientes portadores de DPOC e limitação ao fluxo aéreo. A PEEPi atua como uma sobrecarga para o esforço inspiratório e é descrita como a maior responsável pelo aumento do trabalho respiratório nessa situação ${ }^{24}$. Em pacientes não hipoxêmicos, sedados, em ventilação controlada e bem adaptados, o uso de PEEPe não apresenta benefício clínico, ainda que haja presença de PEEPi ${ }^{12}$. Porém, assim como a sensibilidade para o disparo do ciclo ventilatório é ajustada no máximo, mesmo em pacientes passivamente ventilados, para garantir que não ocorra desconforto quando a atividade inspiratória espontânea ocorrer, o nível de PEEP externo deve ser ajustado durante a fase inicial de ventilação passiva do paciente com DPOC, com o mesmo objetivo. O uso de PEEPe em portadores de DPOC possui suporte teórico de alguns estudos que demonstraram redução do trabalho respiratório sem aumento da hiperinsuflação ${ }^{22,25-30}$. Em casos selecionados, quando estiver difícil o ajuste do volume-minuto, devido à presença de importante hiperinsuflação pulmonar, valores de PEEPe mais baixos e mesmo superiores à PEEPi podem ser testados. Nessa condição especial, o que se procura é uma resposta de desinsuflação. Somente um trabalho demonstrou isso de forma inequívoca, sendo este efeito descrito como uma resposta paradoxal à aplicação da PEEP. Contudo tal efeito foi observado em um pequeno número de pacientes, não tendo sido possível identificar qualquer parâmetro capaz de predizer tal resposta ${ }^{17}$. De qualquer modo, a desinsuflação induzida pela PEEP pode ser detectada pela queda da pressão de platô com o paciente no modo volume controlado ou pelo aumento do volume-corrente no modo pressão controlada.

\section{AJUSTE DA VENTILAÇÃO MECÂNICA NA FASE DE TRANSIÇÃO PARA O DESMAME}

Nessa fase, é imprescindível se otimizar a interação entre paciente-ventilador visando, de um lado, a evitar a atrofia e o descondicionamento muscular respiratório e, do outro, a prevenir o desenvolvimento de fadiga muscular, o que poderia levar a um retardo na liberação do paciente do suporte ventilatório. Devese lembrar da importância da utilização de PEEPe (em 
valores de aproximadamente $85 \%$ do PEEPi) nessa fase do suporte ventilatório de pacientes com DPOC, visando à redução do trabalho inspiratório, através da redução da diminuição relativa de pressão nas vias aéreas necessária para disparar o ventilador ${ }^{26,30}$. Essa estratégia pode, inclusive, melhorar a tolerância do paciente às tentativas de respiração espontânea realizadas durante o processo de liberação da ventilação mecânica ${ }^{31}$.

\section{VENTILAÇÃO COM PRESSÃO DE SUPORTE}

Recomendação: A ventilação com pressão de suporte (PSV) parece ser o melhor modo ventilatório para essa fase de suporte parcial. O nível de suporte pressórico deve corresponder ao menor necessário para manter a freqüência respiratória entre 20 e 30 irpm. Este objetivo é comumente atingido com 15 a 20 $\mathrm{cmH}_{2} \mathrm{O}$ de pressão de suporte, mas existem grandes variações interindividuais.

\section{Grau de Recomendação: D}

Comentário: Na fase de suporte ventilatório parcial, é importante que o retorno da carga para a musculatura respiratória seja realizado de forma controlada. $\mathrm{O}$ uso de níveis adequados de PSV pode ser útil para manter atividade diafragmática, evitando sua fadiga ${ }^{32}$. Existe uma relação inversa entre valores de PSV e valores de volume-corrente, freqüência respiratória e da relação $\mathrm{f} / \mathrm{VT}^{33}$. Entretanto, não há grandes variações nesses parâmetros com valores de PSV entre 5 e $10 \mathrm{cmH}_{2} \mathrm{O}$ ${ }^{34}$. Não existem, porém, estudos avaliando especificamente o uso da PSV em ventilação invasiva, na fase de suporte parcial, em portadores de DPOC. Altos níveis de pressão de suporte podem comprometer a interação entre paciente-ventilador mecânico. Em um estudo foi encontrado que a PSV efetivamente reduziu o trabalho respiratório inspiratório, porém aumentou o trabalho expiratório. Com uma pressão de suporte de $20 \mathrm{cmH}_{2} \mathrm{O}$, o início do esforço expiratório precedeu a cessação do fluxo inspiratório em 5 de 12 pacientes ${ }^{35}$. O ajuste do critério de ciclagem da PSV, disponibilizado em alguns ventiladores modernos, pode, potencialmente, evitar essa interação adversa.

\section{VENTILAÇÃO ASSISTIDA PROPORCIONAL}

Recomendação: Não há evidências que demonstrem superioridade da ventilação assistida proporcional (PAV) em relação à ventilação com pressão de suporte (PSV) quanto a desfechos clínicos relevantes para pacientes com DPOC em ventilação invasiva.

\section{Grau de Recomendação: D}

Comentário: A PAV oferta suporte ventilatório através de ajustes que geram ganho de fluxo e de volume, tendo por base a elastância (inverso da complacência) do sistema respiratório e a resistência de vias aéreas do paciente e em proporção ao esforço muscular realizado a cada ciclo respiratório. Tem, portanto, o potencial de oferecer um suporte ventilatório individualizado, ciclo a ciclo, resultando em maior sincronia, melhorando a interação entre paciente-ventilador e diminuindo o número de esforços musculares ineficazes, isto é, aqueles que não conseguem disparar o ventilador, fato possível quando se utilizam altos valores de pressão de suporte em pacientes com DPOC. Por outro lado, os ajustes do ventilador na PAV são mais complexos e há o risco de fenômenos de runaway (perda de controle) sempre que os ajustes de ganho superam a demanda e a impedância do sistema respiratório. Seus resultados sobre o trabalho respiratório, conforto e drive respiratório variam em função do grau de suporte programado ${ }^{36-39}$.

\section{OUTROS TRATAMENTOS}

\section{MISTURA HÉLIO-OXIGÊNIO (HELIOX)}

Recomendação: A aplicação de Heliox a pacientes com DPOC intubados pode resultar em diminuição da auto-PEEP e do trabalho respiratório. Contudo, não há evidências quanto a desfechos clínicos relevantes para recomendar seu uso rotineiro neste contexto clínico.

\section{Grau de Recomendação: D}

Comentário: A mistura hélio-oxigênio (Heliox) tem uma densidade inferior à mistura de ar: oxigênio-nitrogênio e seu uso reduz a resistência de vias aéreas, promovendo um fluxo de padrão laminar. Estudos controlados têm demonstrado que a sua aplicação não apenas reduz a resistência de vias aéreas, mas também o aprisionamento de ar, a auto-PEEP, as pressões nas vias aéreas e pode atenuar as repercussões hemodinâmicas da hiperinsuflação dinâmica como hipotensão, baixo débito cardíaco e pulso paradoxal ${ }^{40,41}$. Além disso, sua aplicação pode diminuir o trabalho respiratório na fase de desmame da ventilação mecânica ${ }^{42}$. Apesar de relativamente simples quando administrado por máscara, heliox é tecnicamente difícil de usar durante a ventilação mecânica invasiva, devido às influências no fluxo de gás e volume-corrente medidos. Seu valor para o tratamento da DPOC descompensada é, portanto, ainda questionável, difícil e dispendioso. 


\section{FLUXOGRAMA}

\section{Paciente com DPOC intubado em VM}

\section{Ł}

Tratamento da causa básica da exacerbação

Otimização do tratamento da DPOC
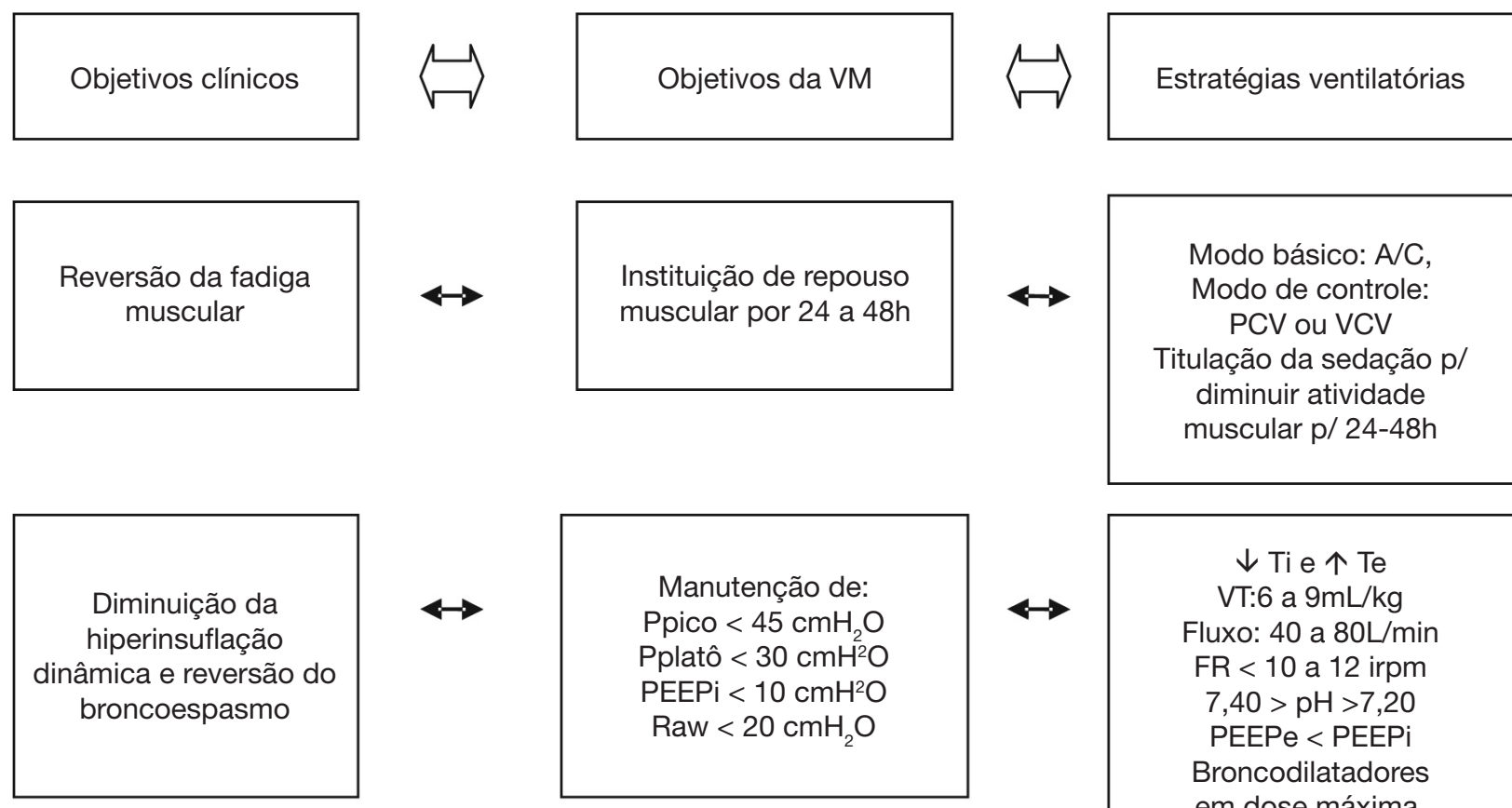

\begin{tabular}{|c|}
$\downarrow$ \\
$\downarrow$ Ti e $\uparrow \mathrm{Te}$ \\
VT:6 a $9 \mathrm{~mL} / \mathrm{kg}$ \\
Fluxo: 40 a $80 \mathrm{~L} / \mathrm{min}$ \\
FR $<10$ a $12 \mathrm{irpm}$ \\
$7,40>\mathrm{pH}>7,20$ \\
PEEPe $<$ PEEPi \\
Broncodilatadores \\
em dose máxima.
\end{tabular}
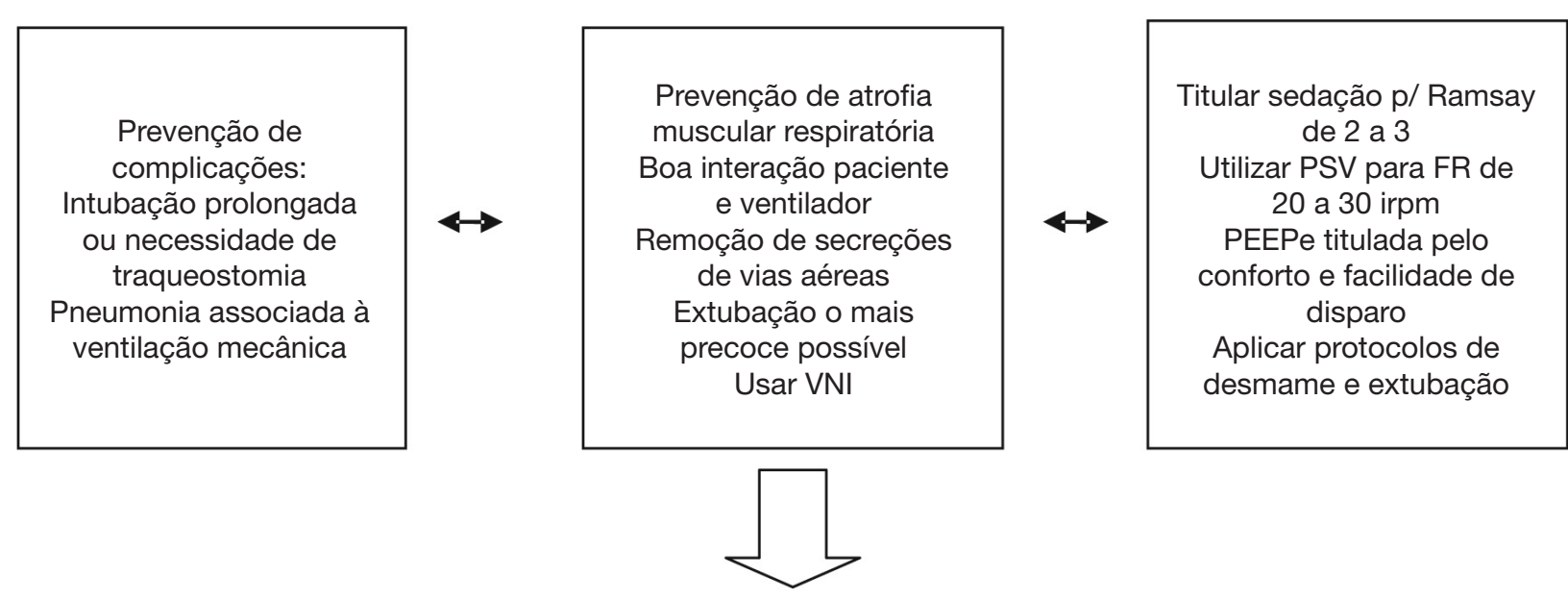
Encaminhamento para centro de referência
após alta 


\section{REFERÊNCIAS}

01. II Consenso Brasileiro sobre Doença Pulmonar Obstrutiva Crônica. J Bras Pneumol, 2004;30:S5.

02. Bach PB, Brown C, Gelfand SE et al - Management of acute exacerbations of chronic obstructive pulmonary disease: a summary and appraisal of published evidence. Ann Intern Med, 2001;134:600-620.

03. Stoller JK - Clinical practice. Acute exacerbations of chronic obstructive pulmonary disease. N Engl J Med, 2002;346:988-994.

04. Raurich JM, Perez J, Ibanez $\mathrm{J}$ et al - In-hospital and 2-year survival of patients treated with mechanical ventilation for acute exacerbation of COPD. Arch Bronconeumol, 2004;40:295-300.

05. Peigang $\mathrm{Y}$, Marini $\mathrm{JJ}$ - Ventilation of patients with asthma and chronic obstructive pulmonary disease. Curr Opin Crit Care, 2002;8:70-76.

06. Mehta S, Hill NS - Noninvasive ventilation in acute respiratory failure. Respir Care Clin N Am, 1996;2:267-292.

07. Hoo GW, Hakimian N, Santiago SM - Hypercapnic respiratory failure in COPD patients: response to therapy. Chest, 2000;117:169-177.

08. Laghi F, D'Alfonso N, Tobin MJ - Pattern of recovery from diaphragmatic fatigue over 24 hours. J Appl Physiol, 1995;79:539-546.

09. Marini J - Controlled Mechanical Ventilation, em: Mancebo JNA, Brochard L - Mechanical Ventilation and Weaning. New York: Springer; 2003.

10. Blanch PB, Jones M, Layon AJ et al - Pressure-preset ventilation. Part 2: Mechanics and safety. Chest, 1993;104:904-912.

11. Blanch PB, Jones M, Layon AJ et al - Pressure-present ventilation. Part 1: Physiologic and mechanical considerations. Chest, 1993;104:590599

12. Blanch L, Bernabe F, Lucangelo $U$ - Measurement of air trapping, intrinsic positive end-expiratory pressure, and dynamic hyperinflation in mechanically ventilated patients. Respir Care, 2005;50:110-124

13. Slutsky AS - Mechanical ventilation. American College of Chest Physicians' Consensus Conference. Chest, 1993;104:1833-1859.

14. Leatherman JW - Mechanical ventilation in obstructive lung disease. Clin Chest Med, 1996;17:577-590.

15. Feihl F, Perret C - Permissive hypercapnia. How permissive should we be? Am J Respir Crit Care Med, 1994;150:(6 Pt 1):1722-1737.

16. Hickling $\mathrm{K}$ - Targets during Mechanical Ventilation, em: Marini J, Slutsky AS - Physiological Basis of Ventilatory Support. New York: Marcel Dekker; 1998;655-695.

17. Caramez MP, Borges JB, Tucci MR et al - Paradoxical responses to positive end-expiratory pressure in patients with airway obstruction during controlled ventilation. Crit Care Med, 2005;33:1519-1528.

18. Cannizzaro G, Garbin L, Clivati A et al - Correction of hypoxia and hypercapnia in COPD patients: effects on cerebrovascular flow. Monaldi Arch Chest Dis, 1997;52:9-12.

19. Vieillard-Baron A, Prin S, Augarde R et al - Increasing respiratory rate to improve $\mathrm{CO} 2$ clearance during mechanical ventilation is not a panacea in acute respiratory failure. Crit Care Med, 2002;30:1407-1412.

20. Guerin C, Lemasson S, La Cara MF et al - Physiological effects of constant versus decelerating inflation flow in patients with chronic obstructive pulmonary disease under controlled mechanical ventilation. Intensive Care Med, 2002;28:164-169.

21. Yang SC, Yang SP - Effects of inspiratory flow waveforms on lung mechanics, gas exchange, and respiratory metabolism in COPD patients during mechanical ventilation. Chest, 2002;122:2096-2104.

22. Georgopoulos D, Mitrouska I, Markopoulou K et al - Effects of breathing patterns on mechanically ventilated patients with chronic obstructive pulmonary disease and dynamic hyperinflation. Intensive Care Med, 1995;21:880-886.

23. Crossley DJ, McGuire GP, Barrow PM et al - Influence of inspired oxygen concentration on deadspace, respiratory drive, and $\mathrm{PaCO} 2$ in intubated patients with chronic obstructive pulmonary disease. Crit Care Med, 1997;25:1522-1526.

24. Coussa ML, Guerin C, Eissa NT et al - Partitioning of work of breathing in mechanically ventilated COPD patients. J Appl Physiol, 1993;75:1711
1719

25. Smith TC, Marini JJ - Impact of PEEP on lung mechanics and work of breathing in severe airflow obstruction. J Appl Physiol, 1988;65:14881499.

26. Ranieri VM, Giuliani R, Cinnella G et al - Physiologic effects of positive end-expiratory pressure in patients with chronic obstructive pulmonary disease during acute ventilatory failure and controlled mechanical ventilation. Am Rev Respir Dis, 1993;147:5-13.

27. Sydow M, Golisch W, Buscher $\mathrm{H}$ et al - Effect of low-level PEEP on inspiratory work of breathing in intubated patients, both with healthy lungs and with COPD. Intensive Care Med, 1995;21:887-895.

28. Guerin C, LeMasson S, de Varax R et al - Small airway closure and positive end-expiratory pressure in mechanically ventilated patients with chronic obstructive pulmonary disease. Am J Respir Crit Care Med, 1997;155:1949-1956.

29. Guerin C, Milic-Emili J, Fournier G - Effect of PEEP on work of breathing in mechanically ventilated COPD patients. Intensive Care Med, 2000;26:1207-1214.

30. Georgopoulos D, Giannouli E, Patakas D - Effects of extrinsic positive end-expiratory pressure on mechanically ventilated patients with chronic obstructive pulmonary disease and dynamic hyperinflation. Intensive Care Med, 1993;19:197-203.

31. Reissmann HK, Ranieri VM, Goldberg P et al - Continuous positive airway pressure facilitates spontaneous breathing in weaning chronic obstructive pulmonary disease patients by improving breathing pattern and gas exchange. Intensive Care Med, 2000;26:1764-1772.

32. Brochard L, Harf A, Lorino $\mathrm{H}$ et al - Inspiratory pressure support prevents diaphragmatic fatigue during weaning from mechanical ventilation. Am Rev Respir Dis, 1989;139:513-521.

33. Tokioka H, Saito S, Kosaka F - Effect of pressure support ventilation on breathing patterns and respiratory work. Intensive Care Med, 1989;15:491-494.

34. Brochard L, Rua F, Lorino $\mathrm{H}$ et al - Inspiratory pressure support compensates for the additional work of breathing caused by the endotracheal tube. Anesthesiology, 1991;75:739-745.

35. Jubran A, Van de Graaff WB, Tobin MJ - Variability of patient-ventilator interaction with pressure support ventilation in patients with chronic obstructive pulmonary disease. Am J Respir Crit Care Med, 1995;152:129136.

36. Passam F, Hoing S, Prinianakis G et al - Effect of different levels of pressure support and proportional assist ventilation on breathing pattern, work of breathing and gas exchange in mechanically ventilated hypercapnic COPD patients with acute respiratory failure. Respiration, 2003;70:355-361.

37. Appendini L, Purro A, Gudjonsdottir $M$ et al - Physiologic response of ventilator-dependent patients with chronic obstructive pulmonary disease to proportional assist ventilation and continuous positive airway pressure. Am J Respir Crit Care Med, 1999;159:(5 Pt 1):1510-1517.

38. Ye Q, Wang C, Tong Z et al - Proportional assist ventilation: methodology and therapeutics on COPD patients compared with pressure support ventilation. Chin Med J (Engl), 2002;115:179-183.

39. Wrigge $\mathrm{H}$, Golisch W, Zinserling $\mathrm{J}$ et al - Proportional assist versus pressure support ventilation: effects on breathing pattern and respiratory work of patients with chronic obstructive pulmonary disease. Intensive Care Med, 1999;25:790-798.

40. Lee DL, Lee H, Chang HW et al - Heliox improves hemodynamics in mechanically ventilated patients with chronic obstructive pulmonary disease with systolic pressure variations. Crit Care Med, 2005;33:968-973.

41. Tassaux D, Gainnier M, Battisti $A$ et al - Helium-oxygen decreases inspiratory effort and work of breathing during pressure support in intubated patients with chronic obstructive pulmonary disease. Intensive Care Med, 2005;31:1501-1507.

42. Diehl JL, Mercat A, Guerot $\mathrm{E}$ et al - Helium/oxygen mixture reduces the work of breathing at the end of the weaning process in patients with severe chronic obstructive pulmonary disease. Crit Care Med, 2003;31:1415-1420. 